\title{
False-Negative CSF Cryptococcal Antigen with Cryptococcus gattii Meningoencephalitis in Southeastern United States: A Case Report and Literature Review
}

\author{
Shawn Esperti (D), Austen Stoelting, Andrew Mangano, Dveet Patel, Jilian Sansbury, \\ and Robert Sherertz
}

Department of Internal Medicine, Grand Strand Health, Myrtle Beach, SC, USA

Correspondence should be addressed to Shawn Esperti; shawn.esperti@hcahealthcare.com

Received 16 March 2020; Revised 20 June 2020; Accepted 29 June 2020; Published 14 July 2020

Academic Editor: Gloria Taliani

Copyright ( $\odot 2020$ Shawn Esperti et al. This is an open access article distributed under the Creative Commons Attribution License, which permits unrestricted use, distribution, and reproduction in any medium, provided the original work is properly cited.

\begin{abstract}
A 70-year-old immunocompetent male in South Carolina was admitted secondary to altered mental status and headache without focal neurological deficits. Head CT was negative. Lumbar puncture (LP) revealed normal glucose, elevated protein, and lymphocytosis. Opening pressure was $15 \mathrm{~cm}$ of H20. CSF lateral flow assay was negative for cryptococcal antigen; CSF cultures showed no growth. The patient rapidly improved on acyclovir and was diagnosed with presumed viral meningitis, as viral PCR and fungal culture were pending at time of discharge. The patient's condition quickly worsened and the patient returned one day later with right arm weakness and dysarthria. Brain MRI revealed T2/flair signal abnormalities in the left frontal lobe with associated parenchymal enhancement. Repeat LP revealed increasing white blood cell count with a worsening lymphocytosis and decreasing glucose, and opening pressure remained normal. CSF fungal culture from the first admission grew Cryptococcus gattii, and repeated CSF cryptococcal antigen and culture returned positive. The patient was started on IV steroids, induction Amphotericin and Fluconazole, followed by maintenance oral Fluconazole. The patient's clinical course was complicated by a brainstem lacunar infarction, which led to demise. We present this case of Cryptococcus gattii meningoencephalitis to highlight the risk factors, characteristics, and challenges in diagnosis and treatment of an emerging disease in the Southeastern United States.
\end{abstract}

\section{Introduction}

Cryptococcal infections have been well recognized as a serious infection of primarily immunocompromised patients worldwide with mortality rates up to $40 \%$ [1]. Cryptococcal organisms are often present in soil and infect the host through inhalation of spores with subsequent dissemination from the lungs to the brain [2]. Most cases of cryptococcal meningitis have been presumed Cryptococcus neoformans, as Cryptococcus gattii is rarely reported and historically only endemic to tropical regions [3]. Before 1999, clinical isolates of C. gattii in North America were almost nonexistent with a small number of cases reported in California and Hawaii [4]. Since 2004, multiple cases of human C. gattii infection have emerged in Oregon, associated with an outbreak on Vancouver Island and in mainland British Columbia, Canada
[5]. Since this outbreak from 2005 to January 2013, 169 human cases of C. gattii infections were reported to the CDC with most confirmed cases in Oregon (88), Washington (31), California (28), Georgia (8), Florida (3), and one case each from Alabama, Colorado, Rhode Island, South Carolina, and Utah [6]. Despite the emerging number of cases, successful diagnosis and recognition remains a challenge. Historically, cryptococcal infections have been associated with HIV-infected patients, thus successful diagnosis has been especially challenging in immunocompetent patients. In addition, less is known about C. gattii infections compared to C. neoformans, and its incidence is likely underreported.

In suspected patients, the mainstay of diagnosis of Cryptococcus has become antigen immunoassays as they are highly sensitive, specific, and result quickly, while fungal cultures take days to weeks to grow [7]. The most widely 
used assays include the lateral flow assay, latex agglutination, and enzyme immunoassays. Lateral flow assays (LFA) are the most widely used due to objectivity, affordability, and turnover time [8]. A positive LFA is followed by the more labor intensive latex agglutination (LA) or enzyme immunoassay (EIA) to determine infection titers. Our lab used Immuno-Mycologics (IMMY) LFA, followed by Latex Agglutination System (CALAS; Meridian Biosciences). Despite such high sensitivity and specificity quoted of $>99 \%$ in detecting CSF antigen for both the IMMY LFA and LA (CALAS; Meridian Biosciences), false-negative results have been reported [9-11]. We present this case of initial falsenegative CSF cryptococcal antigen with Cryptococcus gattii meningoencephalitis to highlight the risk factors, characteristics, and challenges in diagnosis and treatment of an emerging disease in the Southeastern United States. We will also examine current antigen immunoassays used, pitfalls, and phenomena that can lead to rarely reported falsenegative results with resultant delayed diagnosis and treatment.

\section{Case}

A 70-year-old male with a past medical history of low testosterone, hypertension, benign prostatic hyperplasia, and no known travel history presented with confusion and headache in South Carolina. The patient had been recently treated for community-acquired pneumonia and completed a 5-day course of amoxicillin/clavulanic acid as an outpatient. He presented 5 days later after developing a frontal headache and short-term memory deficits. Vital signs were significant for a fever of 100.4. Physical exam revealed lethargy without any focal neurological deficits. A CT scan of the head was normal. Lumbar puncture showed a cerebrospinal fluid (CSF) WBC of $103 \mathrm{~K} / \mathrm{mm}^{3}$ with a differential of $55 \%$ lymphocytes, $7 \%$ neutrophils, $8 \%$ monocytes, glucose $56 \mathrm{mg} / \mathrm{dL}$, and protein $180 \mathrm{mg} / \mathrm{dL}$. Opening pressure was $15 \mathrm{~cm} \mathrm{H}_{2} \mathrm{O}$. The patient was started on vancomycin, ceftriaxone, ampicillin, and acyclovir for empiric treatment of meningitis and encephalitis. CSF studies were negative for cryptococcal antigen, Lyme IgM antibody, Toxoplasmosis IgG antibody, varicella, VDRL, and CMV. Viral HSV PCR was pending and bacterial gram stain, culture, and fungal culture revealed no growth on day 3 of hospitalization. The patient rapidly improved and was discharged with suspected viral meningitis on acyclovir.

The patient returned to the hospital one day after discharge with new onset right-sided weakness and dysarthria. MRI of the brain revealed T2/flair signal abnormalities in the left frontal lobe with associated parenchymal enhancement (refer to Figure 1). Repeat LP was performed and CSF showed a WBC of $621 \mathrm{mg} / \mathrm{dL}$ with a differential of 85\% lymphocyte, 29\% PMNs, 16\% monocytes, CSF glucose $21 \mathrm{mg} / \mathrm{dL}$, and CSF protein $127 \mathrm{mg} / \mathrm{dL}$. Opening pressure was $19 \mathrm{~cm} \mathrm{H}_{2} \mathrm{O}$. CSF fungal cultures from the previous admission grew Cryptococcus gattii after 5 days. HIV and hepatitis $\mathrm{B}$ and $\mathrm{C}$ were negative; serum IgA, IgM, and IgG levels and ANA were normal. Repeat CSF antigen was obtained but not resulted, and gram stain and bacterial cultures showed no growth. Fungal cultures grew C. gattii in 3 days. The patient was started on induction therapy of IV liposomal Amphotericin and Fluconazole for four weeks. A third LP was performed after two weeks of induction therapy with LFA-confirmed positive cryptococcal antigen and a titer of $1: 2560$ on LA. There was no growth on fungal culture. Repeat weekly LPs showed decreasing CSF antigen to $1: 80$ titers and continued sterile fungal cultures. The patient was transitioned and discharged on oral Fluconazole for maintenance therapy. Ten days later, the patient returned to the hospital after suffering a large brainstem lacunar stroke (refer to Figure 2). He was transitioned to inpatient hospice and later expired.

\section{Discussion}

Since the British Columbia outbreak in 1999, the C. gattii has become more prevalent in the United States with the CDC reporting cases in Oregon, Washington, California, Georgia, Florida, Alabama, Colorado, Rhode Island, South Carolina, and Utah [6]. Despite the recent emergence of C. gattii, infections from this species are still likely underreported compared to C. neoformans. While Cryptococcus more often infects immunocompromised patients, C. gattii has shown to have a much stronger affinity to affect immunocompetent patients [3]. Many centers have identified an increasing number (up to 20\%) of cases of Cryptococcus in immunocompetent patients [12]. Clinicians should be suspicious of patients presenting with signs of meningitis who are from potentially endemic regions such as the Southeastern United States or those who have traveled to these areas.

Excluding HIV, other comorbid conditions have been associated with increased risk of C. gattii infections. Immunocompromised hosts include those with solid organ transplants, hematologic malignancies, other malignancies, rheumatic diseases, and those receiving immunosuppressive therapies including corticosteroids and TNF-alpha inhibitors [13]. Immunocompetent hosts at the highest risk are patients with chronic diseases such as cirrhosis, chronic lung disease, chronic renal insufficiency, sarcoidosis, and diabetes mellitus. Once C. gattii infection has been established in an HIV-negative patient without the aforementioned risk factors, less common forms of immunosuppression should be considered and evaluated if feasible. C. gattii infection has been associated with patients with autoantibodies to GM-CSF, hyper IgM/IgE disorders, lymphoproliferative disorders, CD4+ T-cell lymphocytopenia, and usage of monoclonal antibodies, specifically infliximab, etanercept, adalimumab, and alemtuzumab [14].

Prompt recognition and treatment of central nervous system infections related to C. gattii is crucial, as there are several potentially devastating neurological complications. The most common is elevated intracranial pressure and hydrocephalus [15]. Opening pressure should be obtained at initial diagnostic LP and at two weeks when repeat fungal cultures are obtained [16]. If the patient develops signs and symptoms of elevated intracranial pressure, serial LPs and ventriculoperitoneal shunt may be indicated [17, 18]. A complication of chronic cerebral meningitis is lacunar cerebral infarctions. Infection can lead to vasculitis, 


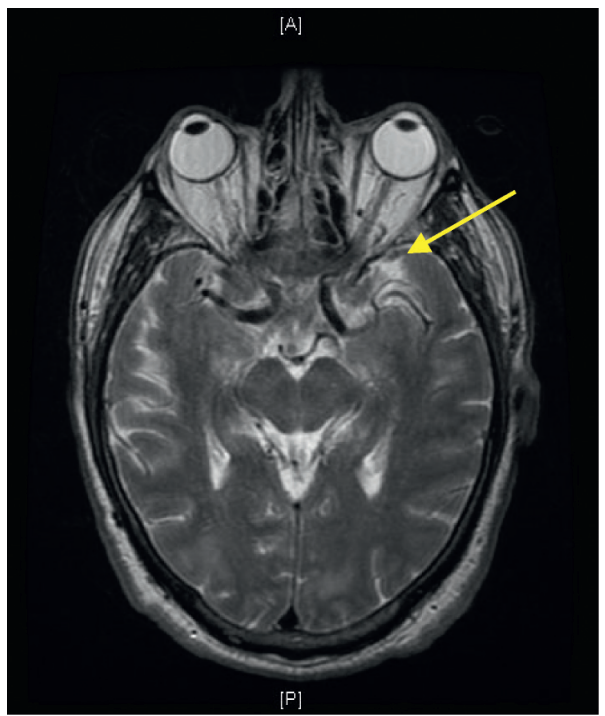

FIGURE 1: MRI brain with and without contrast showing diffuse leptomeningeal enhancement and several scattered foci of restricted diffusion, particularly in the left frontal and parietal lobes. There are some suggestions of T2/flair signal abnormality in the left frontal lobe with associated enhancement of the parenchyma, which could suggest early cerebritis (yellow arrow).

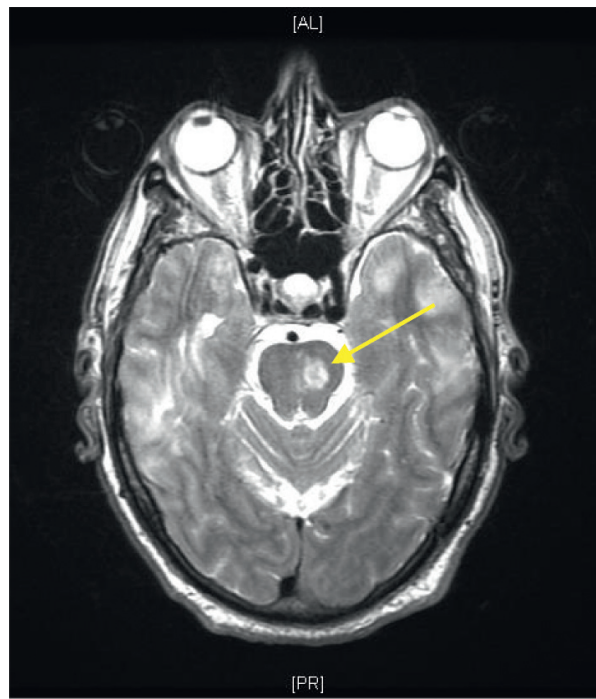

(a)

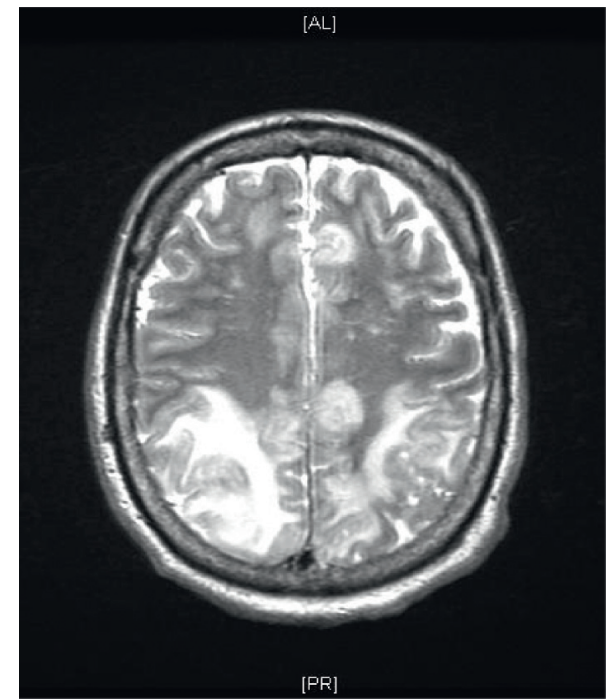

(b)

FIGURE 2: MRI brain with and without contrast showing (a) new left brain stem lacunar infarct (yellow arrow) and (b) worsening sulcal and cisternal T2 hyperintensity and nodular pial enhancement suggesting progression of meningitis. New rather extensive symmetric vasogenic edema due to progressive cerebritis.

inflammation-induced vasospasms, and thrombosis presenting as lacunar infarctions often in the thalamus, internal capsule, basal ganglia, and rarely the brainstem, as occurred in this patient [19].

The LFA, LA, and EIA have been shown to have sensitivities and specificities as high as $100 \%$ in detecting cryptococcal antigen in the CSF [20]. A recent study using 51 archived CSF samples compared the performance detection of 4 immunoassays: Meridian EIA, LA test, IMMY EIA, and IMMY LFA. Among the 51 CSF samples, 18 were positive and 33 were negative and the results showed $100 \%$ agreement among positive (18/18) and negative (33/33) samples for each assay [20]. Despite the success of immunoassays have had in detecting Cryptococcus CSF antigen, data on C. gattii immunoassays are minimal and sample size is small. False-negative testing with the IMMY LFA assay from the prozone effect has been documented $[20,21]$. The prozone effect is often observed in the presence of a high antigen titer as in this patient who had titers of $1: 2560$ after two weeks of treatment [22]. When a large antibody response is mounted, antibodies bind to the cryptococcal antigen, leaving no antigen for the IA to detect, resulting in a false-negative test [22]. In addition, low fungal burden in localized diseases such as isolated cryptococcoma and acapsular cryptococcal variants can lead to false-negative antigen tests, thus delaying diagnosis [23]. In this case, delayed diagnosis may 
have been avoided if the LFA or LA were repeated rather than anchoring on viral meningitis. Due to pitfalls and potential false-negative results of immunoassays, clinicians should consider repeat cryptococcal antigen testing in both immunocompromised and immunocompetent individuals in endemic areas.

\section{Conclusion}

Cryptococcus gattii infection should be highly considered in patients presenting with meningitis who reside in or have traveled in geographic areas with known cases, even in the absence of underlying immunosuppression. Despite the success immunoassays have had in detecting Cryptococcus CSF antigen, data on C. gattii immunoassays are minimal. False negative detections, as in our patient's case, can lead to delayed diagnosis and poor outcomes. This case highlights the risk factors, characteristics, and challenges in diagnosis and treatment of Cryptococcus gattii meningoencephalitis in the Southeastern United States.

\section{Data Availability}

No data were used to support this study.

\section{Consent}

Written consent was obtained and is available upon request.

\section{Disclosure}

The views expressed in this publication represent those of the authors and do not necessarily represent the official views of HCA Healthcare or any of its affiliated entities.

\section{Conflicts of Interest}

The authors declare that they have no conflicts of interest.

\section{Acknowledgments}

This research was supported (in whole or in part) by HCA Healthcare and/or an HCA Healthcare affiliated entity.

\section{References}

[1] E. Pasquier, J. Kunda, P. De Beaudrap et al., "Long term mortality and disability in Cryptococcal Meningitis: a systematic literature review," Clinical Infectious Diseases, vol. 66, no. 7, pp. 1122-1132, 2017.

[2] N. M. Walsh, M. R. Botts, A. J. Mcdermott et al., "Infectious particle identity determines dissemination and disease outcome for the inhaled human fungal pathogen Cryptococcus," PLOS Pathogens, vol. 15, no. 6, Article ID e1007777, 2019.

[3] S. C.-A. Chen, W. Meyer, and T. C. Sorrell, "Cryptococcus gattii infections," Clinical Microbiology Reviews, vol. 27, no. 4, pp. 980-1024, 2014.

[4] J. R. Harris, S. R. Lockhart, G. Sondermeyer et al., "Cryptococcus gattii infections in multiple states outside the US Pacific Northwest," Emerging Infectious Diseases, vol. 19, no. 10, pp. 1620-1626, 2013.
[5] L. MacDougall, S. E. Kidd, E. Galanis et al., "Spread ofCryptococcus gattiiin British Columbia, Canada, and detection in the pacific northwest, USA," Emerging Infectious Diseases, vol. 13, no. 1, pp. 42-50, 2007.

[6] Centers for Disease Control and Prevention (CDC), "Emergence of Cryptococcus gattii-pacific northwest, 2004-2010," MMWR. Morbidity and Mortality Weekly Report, vol. 59, no. 28 , pp. $865-868,2010$.

[7] N. Navabi, M. Montebatsi, M. Scott, S. J. Gluckman, and M. J. A. Reid, "Case report," Journal of the International Association of Providers of AIDS Care (JIAPAC), vol. 14, no. 2, pp. 123-126, 2015.

[8] K. M. Koczula and A. Gallotta, "Lateral flow assays," Essays in Biochemistry, vol. 60, no. 1, pp. 111-120, 2016.

[9] E. Mpoza, L. Mukaremera, D. A. Kundura et al., "Evaluation of a point-of-care immunoassay test kit "StrongStep" for cryptococcal antigen detection," PLoS One, vol. 13, no. 1, Article ID e0190652, 2018.

[10] D. H. Cáceres, A. Zuluaga, Á. M. Tabares, T. Chiller, Á. González, and B. L. Gómez, "Evaluation of a Cryptococcal antigen lateral flow assay in serum and cerebrospinal fluid for rapid diagnosis of cryptococcosis in Colombia," Revista do Instituto de Medicina Tropical de Sao Paulo, vol. 59, p. e76, 2017.

[11] N. Kojima, M. Chimombo, and D. G. Kahn, "False-negative cryptococcal antigen test due to the postzone phenomenon," Aids, vol. 32, no. 9, pp. 1201-1202, 2018.

[12] P. G. Pappas, "Cryptococcal infections in non-HIV-infected patients," Transactions of the American Clinical and Climatological Association, vol. 124, pp. 61-79, 2013.

[13] J. Beardsley, T. C. Sorrell, and S. C.-A. Chen, "Central nervous system cryptococcal infections in non-HIV infected patients," Journal of Fungi, vol. 5, no. 3, p. 71, 2019.

[14] E. K. Maziarz and J. R. Perfect, "Cryptococcosis," Infectious Disease Clinics of North America, vol. 30, no. 1, pp. 179-206, 2016.

[15] E. Galanis, L. Hoang, P. Kibsey, M. Morshed, and P. Phillips, "Clinical presentation, diagnosis and management of Cryptococcus gattiiCases: lessons learned from British Columbia," Canadian Journal of Infectious Diseases and Medical Microbiology, vol. 20, no. 1, pp. 23-28, 2009.

[16] M. Abassi, D. R. Boulware, and J. Rhein, "Cryptococcal meningitis: diagnosis and management update," Current Tropical Medicine Reports, vol. 2, no. 2, pp. 90-99, 2015.

[17] M. Rigi, S. J. Almarzouqi, M. L. Morgan, and A. G. Lee, "Papilledema: epidemiology, etiology, and clinical management," Eye and Brain, vol. 7, pp. 47-57, 2015.

[18] D. Vela-Duarte, E. Nyberg, S. Sillau et al., "Lacunar stroke in cryptococcal meningitis: clinical and radiographic," Journal of Stroke and Cerebrovascular Diseases, vol. 28, no. 6, pp. 1767-1772, 2019.

[19] S. K. Yadava and T. Fazili, "Postzone phenomenon resulting in a false-negative cerebral spinal fluid cryptococcal antigen lateral flow assay," Aids, vol. 33, no. 6, pp. 1099-1100, 2019.

[20] M. J. Binnicker, D. J. Jespersen, J. E. Bestrom, and L. O. Rollins, "Comparison of four assays for the detection of cryptococcal antigen," Clinical and Vaccine Immunology, vol. 19, no. 12, pp. 1988-1990, 2012.

[21] G.-H. Lee, I. Arthur, and M. Leung, "False-negative serum cryptococcal lateral flow assay result due to the prozone phenomenon," Journal of Clinical Microbiology, vol. 56, no. 4, 2018.

[22] R. Sidana, H. Mangala, S. Murugesh, and K. Ravindra, "Prozone phenomenon in secondary syphilis," Indian Journal 
of Sexually Transmitted Diseases and AIDS, vol. 32, no. 1, pp. 47-49, 2011.

[23] M. A. S. B. Borges, J. A. D. Araújo Filho, R. D. B. A. Soares, J. E. Vidal, and M. D. Turchi, "False-negative result of serum cryptococcal antigen lateral flow assay in an HIV-infected patient with culture-proven cryptococcaemia," Medical Mycology Case Reports, vol. 26, pp. 64-66, 2019. 